\title{
Emergent Phase Coherence of Stripe Order in Manganites Revealed with Cryogenic Scanning Transmission Electron Microscopy
}

Ismail El Baggari ${ }^{1}$, Benjamin H. Savitzky ${ }^{1}$, Robert Hovden ${ }^{2}$, Alemayehu S. Admasu ${ }^{3}$, Jaewook Kim ${ }^{3}$, Sang-Wook Cheong ${ }^{4}$ and Lena F. Kourkoutis ${ }^{2,5}$

1. Department of Physics, Cornell University, Ithaca NY, USA.

2. School of Applied and Engineering Physics, Cornell University, Ithaca NY, USA.

3. Department of Physics and Astronomy, Rutgers University, Piscataway NJ, USA.

${ }^{4 .}$ Rutgers Center for Emergent Materials, Rutgers University, Piscataway NJ, USA.

${ }^{5}$ Kavli Institute at Cornell, Cornell University, Ithaca NY, USA.

Low temperature phase diagrams of strongly correlated systems reveal complexity and competition between mismatched orders, best exemplified in manganites where the inhomogeneous coexistence of metallic domains with charge-ordered patches results in colossal magnetoresistance [1]. Charge order, or stripes, is a prevalent electronic instability where electrons form periodic patterns, breaking lattice symmetry and competing with other phases. Disorder is thought to govern the emergence of the low temperature striped phases, causing, for instance, a tendency for gradual crossover rather than sharp phase transitions [1]. Real space visualization of the evolution of stripe order promises a deeper understanding of the onset of ordered phases.

Dark-field transmission electron microscopy has allowed direct observation of stripes in manganites with nanometer resolution [2]. In those studies, contrast modulation is thought to originate from the choreographed expansion and contraction of oxygen octahedra due to charge localization and the JahnTeller effect. However, direct, atomic-scale mapping of stripes and their temperature dependence is still lacking. Here we demonstrate atomic-resolution high-angle annular dark field scanning transmission electron microscopy (HAADF STEM) at room and cryogenic temperatures ( 93K), allowing vivid visualization of the evolution of stripes in $\mathrm{Bi}_{1-\mathrm{x}}(\mathrm{Sr}, \mathrm{Ca})_{\mathrm{x}} \mathrm{MnO}_{3}(\mathrm{BSCMO})$, a model charge-ordered system with $T_{c} \sim 300 \mathrm{~K}$. Room temperature and cryogenic HAADF imaging was performed in an aberrationcorrected FEI Titan Themis operating at $300 \mathrm{kV}$, using a $30 \mathrm{mrad}$ convergence angle and $68-340 \mathrm{mrad}$ collection angles. Due to reduced stage stability at cryogenic temperatures, we acquired stacks of 20-30 images using a 1-2 $\mathrm{s}$ /pixel dwell time and applied rigid-registration schemes to align and average frames.

Figure 1(a) shows a HAADF image of BSCMO at $93 \mathrm{~K}$, with the $\mathrm{Bi} / \mathrm{Sr} / \mathrm{Ca}$ (green) and $\mathrm{Mn}$ (red) columns clearly resolved, highlighting resolution and signal-to-noise ratio on par with room temperature imaging. The Fourier transform amplitude of the lattice image contains superlattice peaks decorating lattice Bragg peaks, reflecting the presence of a modulation with $\sim 3$ unit cell periodicity. We map the real space structure of the modulation that gives rise to the observed peaks. As shown in Fig. 1(c,d), stripes originate from picometer scale periodic lattice displacements of the cations. The order parameter (OP) is $\Delta(r)=$ $\mathfrak{R}\{\mathbf{A}(\boldsymbol{r}) \exp [i \phi(\boldsymbol{r})] \exp [i \boldsymbol{q} . \boldsymbol{r}]\}$ where $\boldsymbol{q}$ is the wavevector, $\mathbf{A}(\boldsymbol{r})$ is the amplitude vector, and $\phi(\boldsymbol{r})$ is the phase.

We observe temperature-dependent stripe inhomogeneity, including shear deformations (Fig. 2). To visualize the defect-mediated evolution of stripes, we show in Fig. 3(a,b) coarse-grained $\phi(r)$ fields at $293 \mathrm{~K}$ and $93 \mathrm{~K}$, respectively. The former displays significant phase inhomogeneity including $2 \pi$ phase vortices (dislocations) and large phase gradients (shear). At low temperature, a more uniform, slowly varying phase configuration is observed, indicating emergent phase coherence well below $\mathrm{T}_{\mathrm{c}}$. In Fig. 3(c), 
we plot the correlations of the OP components, notably $\phi(\boldsymbol{r})$ and $|\mathbf{A}(\boldsymbol{r})|$, across temperatures. Amplitude correlations exhibit minimal decay at both temperatures, suggesting that amplitude variations have a negligible effect on stripe evolution. On the other hand, phase correlations decay rapidly at $293 \mathrm{~K}$, becoming uncorrelated after $\sim 5 \mathrm{~nm}$. At $93 \mathrm{~K}$, the phase shows increased homogeneity with correlations remaining finite over accessible length scales $(\sim 10 \mathrm{~nm})$, supporting that emergent phase coherence governs the phenomenology of stripes. In addition to permitting vivid visualization of the emergence of electronic order, cryogenic STEM paves the way for accessing low temperature phases in correlated systems [3].

\section{References:}

[1] E. Dagotto. Science 309 (2005), p. 5732.

[2] S. Mori, C. H. Chen, and S.-W. Cheong. Nature 392 (1998), p. 473.

[3] We acknowledge support from AFOSR (FA 9550-16-1-0305), NSF MRI award (DMR-1429155), and the Packard Foundation. Work made use of CCMR facilities supported by NSF (DMR-1120296).
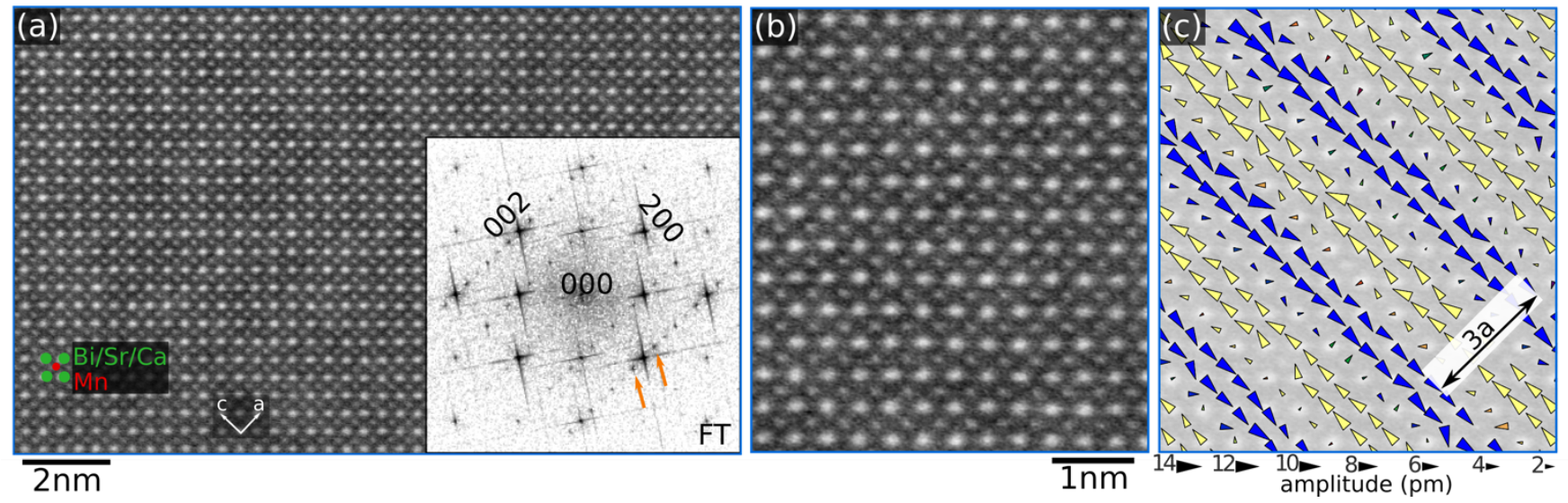

Figure 1. (a),(b) Cryogenic HAADF STEM images of BSCMO with $\mathrm{Bi} / \mathrm{Sr} / \mathrm{Ca}$ and $\mathrm{Mn}$ clearly resolved. The Fourier transform (FT) amplitude exhibits superlattice peaks (orange arrows) in addition to Bragg peaks. (c) Mapping picometer scale displacements (arrows) associated with superlattice peaks. The blue (yellow) arrows correspond to transverse displacements, oriented $90^{\circ}\left(-90^{\circ}\right)$ relative to $\widehat{\boldsymbol{a}}$.

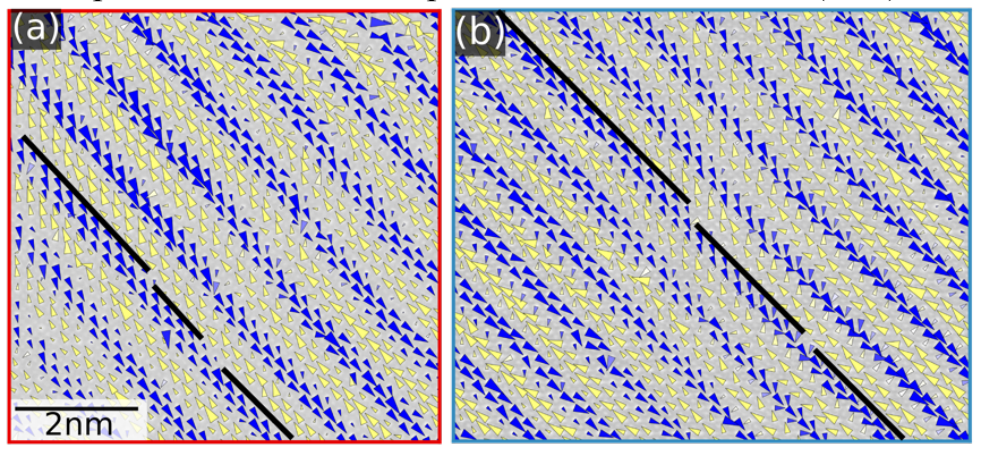

Figure 2. (a),(b) Mapping of shear deformations of stripes at $293 \mathrm{~K}$ and $93 \mathrm{~K}$, respectively. The deformation appears milder and more extended at low temperature.

Figure 3. (a),(b) Phase configuration over a $\sim 40 \mathrm{~nm}$ field of view at $293 \mathrm{~K}$ and $93 \mathrm{~K}$, respectively. Circles correspond to phase vortices (dislocations) and rectangles correspond to shear deformations. (c) Autocorrelations of the phase and amplitude fields at $293 \mathrm{~K}$ and $93 \mathrm{~K}$.

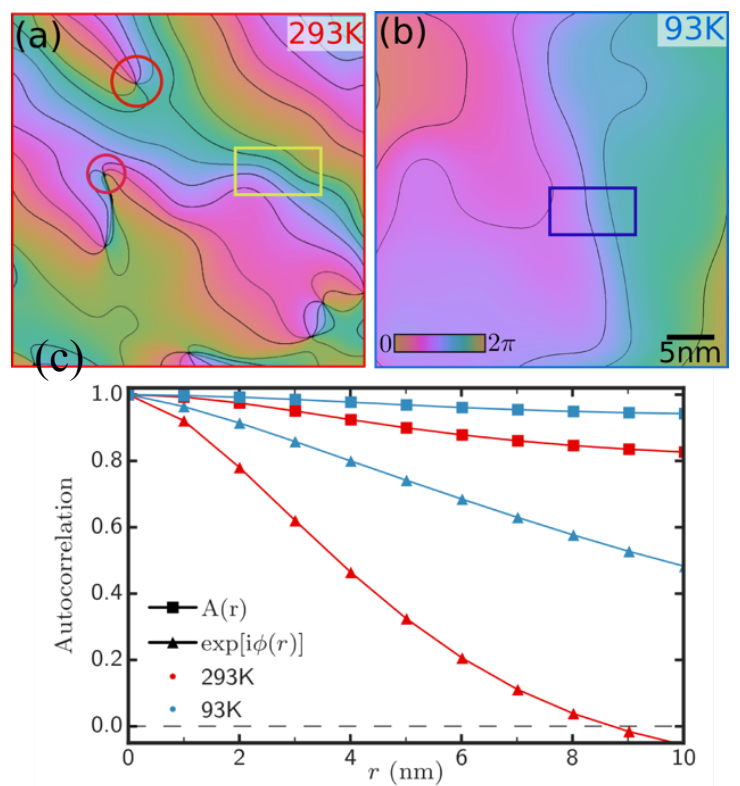

